\title{
Ab Initio Molecular Orbital Calculation of Stabilizing Energy of the Double-Stranded Helix for Isotactic Poly(methyl methacrylate) Large Oligomers
}

\author{
Hiroshi Kusanagi, Yozo Chatani,* and Hiroyuki Tadokoro* \\ Unitika Research Laboratories Inc., Uji, Kyoto 611, Japan \\ * Department of Macromolecular Science, Faculty of Science, Osaka University, \\ Toyonaka, Osaka 560, Japan
}

(Received March 21, 1997)

KEY WORDS Energy Calculation / Ab Initio MO / it-PMMA / Double-Stranded Helix / Stabilizing
Energy /

For the (10/1) double-stranded helix of isotactic poly(methyl methacrylate) (it-PMMA), observed by Xray analysis, ${ }^{1,2}$ we have done theoretical studies on its structural stability. In the first paper, ab initio molecular orbital (MO) calculation was made to evaluate the energetical stability of the double-stranded monomer as a model compound for polymer double-stranded helix. ${ }^{3}$ The monomer model compound was found to be stabilized with intermolecular interactions: MO calculation of the monomer model gave a small stabilizing energy (= the molecular complex formation energy) of $E_{\text {inter }}=$ $0.26 \mathrm{kcal} \mathrm{mol}^{-1}$ of monomer, in comparison with twice isolated monomer. This monomer model is too small as an appropriate model for it-PMMA polymer.

The molecular mechanics (MM) energy calculation method was applied to large oligomer models for itPMMA double-stranded helix. ${ }^{4}$ Large oligomer doublestranded-helices exist as satisfactorily-stable doublestranded helices by large stabilizing energies: for an example, $E_{\text {inter }}(u)=3.1 \mathrm{kcal} \mathrm{mol}^{-1}$ of monomer unit for the double-stranded helix of decamer model. The MM method is easier to deal with large oligomers than the MO method, while potential parameters used in MM calculations of it-PMMA have to be transferred from other molecules and transferability is a troublesome problem. A new energy calculation procedure using the $a b$ initio MO method was derived for estimation of the stabilizing energy on large oligomer double-stranded helices, although the usual $a b$ initio MO method cannot treat polymers. ${ }^{5}$

This procedure was applied to the calculation of the stabilizing energy of the double-stranded helices for it-PMMA large oligomers.

\section{CALCULATION}

\section{Separated Monomer Ab Initio MO Method}

In the separated monomer MO method, ${ }^{5}$ assuming a double-stranded helix composed of two single helices, represented Helix-A and $-\mathrm{B}$, each of which consists of $2 M+1$ monomer units $(-M,-M+1, \cdots,-1,0,1, \cdots$, $M-1, M)$, the stabilizing energy of double-stranded helix $E_{\text {inter }}(u)$ can be obtained by the single summation from the 0 -th monomer unit in the Helix-A to the $k$-th monomer unit in the Helix-B,

$$
\begin{aligned}
E_{\text {inter }}(u) & =[1 /(2 M+1)] E_{\text {inter }} \\
& =[1 /(2 M+1)]\left[(1 / 2) \sum \sum \varepsilon_{\text {inter }}(i, j)\right] \\
& =(1 / 2) \sum \varepsilon_{\text {inter }}(0, k)
\end{aligned}
$$

where $\varepsilon_{\text {inter }}(0, k)$ is the interaction energy between the 0 -th monomer unit in Helix-A and $k$-th monomer unit in Helix-B. In this method, the energy $\varepsilon_{\text {inter }}(0, k)$ is made with each pair of 0 -th monomer unit of Helix-A and $k$-th monomer unit of Helix-B.

$$
\varepsilon_{\text {inter }}(0, k)=\varepsilon_{\mathrm{AB}}^{\mathrm{MO}}(0, k)-\varepsilon_{\mathrm{A}}^{\mathrm{MO}}(0)-\varepsilon_{\mathrm{B}}^{\mathrm{MO}}(k)
$$

where $\varepsilon_{\mathrm{AB}}^{\mathrm{MO}}(0, k)$ is $\mathrm{MO}$ energy of a small super molecule composed of the 0-th monomer in Helix-A and the $k$-th monomer in Helix-B, and $\varepsilon_{\mathbf{A}}^{\mathrm{MO}}(0)$ and $\varepsilon_{\mathbf{B}}^{\mathrm{MO}}(k)$ are the $\mathrm{MO}$ energies of the 0 -th monomer in Helix-A and of the $k$-th monomer in Helix-B, respectively. Since the MO energy is pairwisely calculated for such small super molecules of two monomers, the number of atoms and basis functions do not increase with the oligomer size. Ab initio MO calculation of large oligomer models of it-PMMA double-stranded helix is thus possible.

$A b$ initio MO calculations were carried out using the Hondo- 8 system. The maximal number of atoms is 128 . The 4-31G basis set was adopted, and ab initio SCF-MO method with electron correlation correction by the 2 nd order Moeller-Plesset (MP2) perturbation theory was used for calculation. The present Hondo- 8 program has been used for MP2 calculations involving up to 250 basis functions $(\mathrm{NF}<250)$. The IBM computer work station was used.

\section{Molecular Models of Double-Stranded Helices for It- PMMA Oligomers}

The (10/1) double-stranded helix structure, determined by X-ray analysis, ${ }^{1}$ of it-PMMA was used for the present study. By using one monomer unit geometry of it-PMMA $\left(\mathrm{H}-\mathrm{C}\left(\mathrm{COOCH}_{3}\right)\left(\mathrm{CH}_{3}\right)-\mathrm{CH}_{2}-\mathrm{H}: 17\right.$ atoms $)$, the molecular coordinates of 0 -th monomer unit in Helix-A were constructed as a basis model compound for large oligomer models. The molecular coordinates of 0 -th monomer unit in Helix-B was deduced from that of 0 -th monomer unit in Helix-A according to (10/1) helix symmetry. The same procedure can be applied to the 1st, 2nd, 3rd, $\cdots, k$-th monomer unit in Helix-B. It is thus 
Table I. Monomer MO energy $\left(\varepsilon^{\mathrm{MO}}\right)$ and inter-monomer stabilizing enegy $\left(\varepsilon_{\text {inter }}\right)$ by the separated monomer MO method for the double-stranded helix model of it-PMMA oligomers

\begin{tabular}{|c|c|c|c|c|c|}
\hline \multirow{2}{*}{$k^{\mathrm{a}}$} & $\varepsilon_{\mathbf{A B}}^{\mathrm{MO}}(0, k)^{\mathbf{b}}$ & $\varepsilon_{\mathrm{A}}^{\mathrm{MO}}(0)^{\mathrm{c}}$ & $\varepsilon_{\mathbf{B}}^{\mathrm{MO}}(k)^{\mathrm{d}}$ & $\varepsilon_{\text {inter }}(0, k)^{\mathrm{e}}$ & $\varepsilon_{\text {inter }}(0, k)^{\mathrm{e}}$ \\
\hline & $\mathrm{au}$ & $\mathrm{au}$ & $\mathrm{au}$ & $\mathrm{au}$ & $\mathrm{kcal} \mathrm{mol}^{-1}$ \\
\hline 0 & -690.19170 & -345.09544 & -345.09544 & -0.00082 & -0.515 \\
\hline 1 & -690.19207 & -345.09544 & -345.09539 & -0.00124 & -0.775 \\
\hline 2 & -689.52395 & -345.09544 & -344.42690 & -0.00160 & -1.004 \\
\hline 3 & -690.19168 & -345.09544 & -345.09549 & -0.00076 & -0.475 \\
\hline 4 & -690.19104 & -345.09544 & -345.09545 & -0.00015 & -0.097 \\
\hline 5 & -690.19085 & -345.09544 & -345.09544 & 0.00002 & 0.015 \\
\hline 6 & -690.19094 & -345.09544 & -345.09552 & 0.00002 & 0.012 \\
\hline 7 & -690.19088 & -345.09544 & -345.09546 & 0.00001 & 0.005 \\
\hline 8 & -690.19087 & -345.09544 & -345.09544 & 0.00001 & 0.003 \\
\hline
\end{tabular}

${ }^{a} k$ : Number for indicating the position of a paired monomer in Helix-B. ${ }^{\mathrm{b}} \varepsilon_{\mathrm{AB}}^{\mathrm{MO}}(0, k)$ : MO energy of a small super molecule composed of the 0 -th monomer in Helix-A and the $k$-th monomer in Helix-B. ${ }^{\mathrm{c}} \varepsilon_{\mathrm{A}}^{\mathrm{MO}}(0)$ : MO energy of the 0-th monomer in Helix-A. ${ }^{\mathrm{d}} \varepsilon_{\mathbf{B}}^{\mathrm{MO}}(k)$ : MO energy of the $k$-th monomer in Helix-B. ${ }^{\mathrm{e}} \varepsilon_{\text {inter }}(0, k)$ : Inter-monomer stabilizing energy: $\varepsilon_{\text {inter }}(0, k)=\left[\varepsilon_{\mathbf{A B}}^{\mathrm{MO}}(0, k)-\varepsilon_{\mathbf{A}}^{\mathrm{MO}}(0)-\varepsilon_{\mathbf{B}}^{\mathrm{MO}}(k)\right], \varepsilon_{\text {inter }}(0, k)=\varepsilon_{\text {inter }}(0,-k)$, because of two-fold rotation symmetry normal to helix axis.

Table II. Stabilizing enegy $\left(E_{\text {inter }}\right)$ of the double-stranded helix models of it-PMMA several oligomers

\begin{tabular}{|c|c|c|c|c|c|c|c|c|c|c|c|c|c|c|c|c|c|}
\hline$n^{\mathrm{a}}$ & 1 & 2 & 3 & 4 & 5 & 6 & 7 & 8 & 9 & 10 & 11 & 12 & 13 & 14 & 15 & 16 & 17 \\
\hline$E_{\text {inter }}$ & 0.26 & 0.65 & 1.03 & 1.54 & 2.04 & 2.27 & 2.51 & 2.56 & 2.60 & 2.60 & 2.59 & 2.58 & 2.58 & 2.57 & 2.57 & 2.57 & 2.57 \\
\hline
\end{tabular}

${ }^{a} n$ : Total number of monomers in oligomers, $\mathrm{H}-\left[-\mathrm{C}\left(\mathrm{COOCH}_{3}\right)\left(\mathrm{CH}_{3}\right)-\mathrm{CH}_{2}\right]_{n}-\mathrm{H}$.

possible to calculate $\varepsilon_{\mathrm{AB}}^{\mathrm{MO}}(0, k), \varepsilon_{\mathrm{A}}^{\mathrm{MO}}(0)$, and $\varepsilon_{\mathbf{B}}^{\mathrm{MO}}(k)$ terms in eq 2. Hondo-8 MO program $(\mathrm{NF}<250)$ enables to calculate $\varepsilon_{\mathrm{AB}}^{\mathrm{MO}}(0, k)$ term associated with a pair $(\mathrm{NF}=166)$ of two intertwined monomers, since one monomer unit involves 83 basis functions when the $a b$ initio SCF-MO method is used with the electron correlation correction by means of the 2nd order Moeller-Plesset (MP2) perturbation theory.

\section{RESULTS AND DISCUSSION}

Molecular Weight Dependency of Stabilizing Energy $E_{\text {inter }}$

The separated monomer MO method consists of two calculation steps. In step-1, the inter-monomer stabilizing energy $\varepsilon_{\text {inter }}(0, k)$ is calculated for $k=1,2,3, \cdots M$, according to eq 2 . The calculated $\mathrm{MO}$ energy $\varepsilon_{\mathbf{A B}}^{\mathrm{MO}}(0, k)$, $\varepsilon_{\mathbf{A}}^{\mathrm{MO}}(0), \varepsilon_{\mathbf{B}}^{\mathrm{MO}}(k)$, and inter-monomer stabilizing energy $\varepsilon_{\text {inter }}(0, k)$ are listed in Table I. CPU time for the calculation of each $\varepsilon_{\mathbf{A B}}^{\mathrm{MO}}(0, k)$ term takes about 8 hours. From these $\varepsilon_{\text {inter }}(0, k)$, according to eq $1, E_{\text {inter }}(u)$ was easily calculated for each $M$ (step-2). In eq 1 , the summation on $k$ was cut off at $k=8$, where $\varepsilon_{\text {inter }}(0, k)$ is smaller than $0.003 \mathrm{kcal} \mathrm{mol}^{-1}$. The calculated stabilizing energy $E_{\text {inter }}(u)$ are listed in Table II. We can see the oligomer size dependency of the stabilizing energy $E_{\text {inter }}(u)$. To visualize clearly this situation, the stabilizing energy $E_{\text {inter }}(u)$ was plotted against the number $n$ of monomers in oligomers as shown in Figure 1. With increasing number of $n$, the stabilizing energy $E_{\text {inter }}(u)$ increases rapidly at $M=1-7$, and becomes almost a saturate value of $E_{\text {inter }}(u)=2.6 \mathrm{kcal} \mathrm{mol}^{-1}$ of monomer unit during $M=8-17$. The large molecular-weight effect for the stabilizing energy was confirmed in the energy calculations using the $a b$ initio MO method, in a similar manner as that using the MM method. The double-stranded helix structure of it-PMMA was strongly supported theoretically by the $a b$ initio MO method. The calculation results

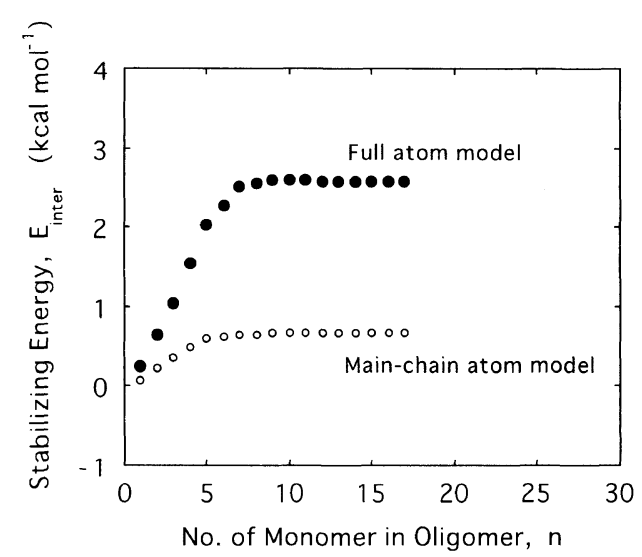

Figure 1. Relation between stabilizing energy $E_{\text {inter }}(u)$ and total number $n$ of monomers in oligomer Helix-B for it-PMMA. $\bigcirc$ indicate values for full atom model in the present study and main-chain atom model in the previous study, ${ }^{4}$ respectively.

for the main-chain atom model $\left[\mathrm{H}-\left(-\mathrm{CH}_{2}-\mathrm{CH}_{2}-\right)_{n}-\mathrm{H}\right]$ of it-PMMA large oligomers ${ }^{5}$ are shown in Figure 1. $E_{\text {inter }}(u)$ display a similar molecular weight dependency and the ratio of the saturated $E_{\text {inter }}(u)=2.6 \mathrm{kcal} \mathrm{mol}^{-1}$ of the full atom model to $E_{\text {inter }}(u)=0.66 \mathrm{kcal} \mathrm{mol}^{-1}$ of the main-chain atom model is 3.94 . That is, for stabilizing the double-stranded helix of it-PMMA large oligomers, the contribution of the side-chain atoms is dominant. The ratio of monomer molecular weight $M_{\text {full }}=\mathrm{C}_{5} \mathrm{H}_{8} \mathrm{O}_{2}=100$ of the full atom model to that $M_{\text {main }}=\mathrm{C}_{2} \mathrm{H}_{4}=28$ of the main-chain atom model is 3.57. Thus the stabilizing energy may be taken to be proportional to the molecular weight of monomer. This proportionality confirms that the stabilizing energy of it-PMMA polymer originates from the Van der Waals interaction. Thus among various intermolecular interactions, i.e., van der Waals interaction, hydrogen-bonding interaction, and electrostatic interaction, only the van 


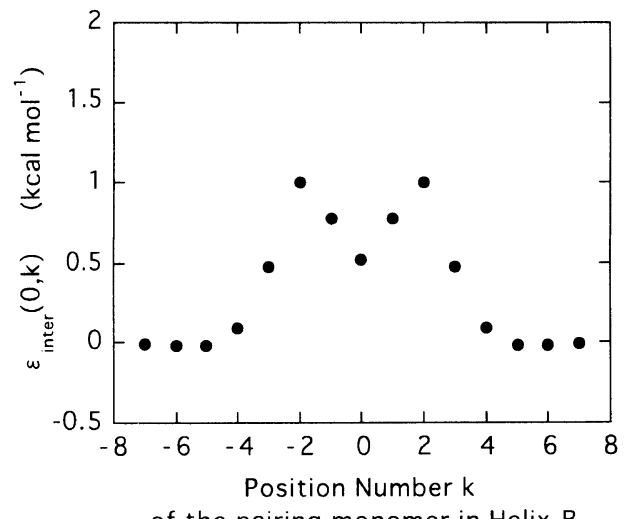

of the pairing monomer in Helix-B

Figure 2. Relation between inter-monomer stabilizing energy $\varepsilon_{\text {inter }}(0, k)$ and number $k$ for indicating the positon of a paired monomer in oligomer Helix-B for it-PMMA full atom model.

der Waals interaction has a fundamental property which the interaction enrgy is closely proportional to the molecular weight of two molecules interacting with each other.

Origin of Stabilizing Energy $E_{\text {inter }}$

It is important to consider the relation between the stabilizing energy $E_{\text {inter }}(u)$ and geometry of doublestranded helix for theoretical analysis of molecular structure of it-PMMA. The constituents of $E_{\text {inter }}(u)$, i.e., the values of inter-monomer stabilizing energy $\varepsilon_{\text {inter }}(0, k)$ were plotted against $k$ as shown in Figure 2. From this figure the relation between inter-monomer stabilizing (cohesive) energy $\varepsilon_{\text {inter }}(0, k)$ and relative position of intertwined two monomers was deduced. That is, a large cohesive energy $\varepsilon_{\text {inter }}(0, k)$ occurs when the two mono- meric units are separated $0-3$ monomeric units along the helix axis and this large energy is produced from the geometry of the helical structure of it-PMMA. Among them, the largest cohesive energy $\varepsilon_{\text {inter }}(0, k)$ occurs when $k=2$, i.e., between the 0 -th monomer unit in Helix-A and the 2nd monomer unit in Helix-B. Thus it is interesting that a set of two monomers separating two monomer units along the helix axis gives larger $\varepsilon_{\text {inter }}(0,2)=1.00 \mathrm{kcal} \mathrm{mol}^{-1}$ than that of two monomers locating at the same monomer height, $\varepsilon_{\text {inter }}(0,0)=0.52$ $\mathrm{kcalmol}^{-1}$. The total stabilizing energy $E_{\text {inter }}(u)$ of double-stranded helix of large oligomers originates from the integration of the van der Waals interaction energies along the helix axis.

From the present MO calculation for large oligomer models of it-PMMA we deduced a conclusion that the long-range intermolecular interaction energies along the helix axis between intertwined two chains may contribute to increasing this stabilizing energy.

Acknowledgment. This work is based in part on results obtained from the MOTECC Package.

\section{REFERENCES}

1. H. Kusanagi, Y. Chatani, and H. Tadokoro, Polymer, 35, 2028 (1994).

2. H. Kusanagi, Polym. J., 28, 708 (1996).

3. H. Kusanagi, Y. Chatani, and H. Tadokoro, Polym. J., 28, 639 (1996).

4. H. Kusanagi, Y. Chatani, and H. Tadokoro, Polym. J., 28, 916 (1996); Macromolecules, 15, 170 (1982).

5. H. Kusanagi, Y. Chatani, and H. Tadokoro, Polym. J., 29, 65 (1997). 